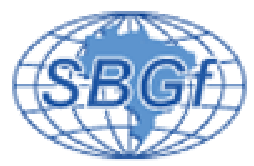

\title{
Utilização de tomografia elétrica na identificação da interface entre uma cobertura antrópica e sedimentos fluviais na área do Sítio Controlado de Geofísica Rasa do IAG/USP
}

Welitom Rodrigues Borges ${ }^{1}$, Jorge Luis Porsani ${ }^{2}$, Elizete M. A. da Silva ${ }^{1}$, Vagner Roberto Elis ${ }^{2}$, Francisco Yukio Hiodo ${ }^{2}$

${ }_{1}^{1}$ Pós-Graduação em Geofísica do Instituto de Astronomia, Geofísica e Ciências Atmosféricas (IAG/USP)

2 Departamento de Geofísica do Instituto de Astronomia, Geofísica e Ciências Atmosféricas (IAG/USP)

Copyright 2004, SBGf - Sociedade Brasileira de Geofísica

Este texto foi preparado para a apresentação no I Simpósio Regional da Sociedade Brasileira de Geofísica São Paulo, 26-28 de revisado pen revrado pela Conissa

\section{Resumo}

Este trabalho mostra os resultados de aquisições de dados de tomografia elétrica 2D, realizadas na área do Sítio Controlado de Geofísica Rasa (SCGR) do IAG, no campus da USP, São Paulo. Os levantamentos foram realizados antes da implantação dos alvos, visando a definição do background elétrico. Os resultados possibilitaram a identificação do limite entre o solo antrópico (aterro) e os sedimentos da planície de inundação do rio Pinheiros.

\section{Introdução}

O aprimoramento dos processos tecnológicos utilizados na implantação de infra-estrutura de centros urbanos evoluiu abruptamente com o passar dos anos. A preocupação com a segurança da população influenciou na implantação de redes subterrâneas de distribuições hidráulicas, energéticas e de comunicação. Com o crescimento da população é necessário o adensamento destas redes, no entanto, devido à falta de comunicação entre as empresas de distribuição, alguns acidentes ocorrem durante a implantação de novas redes, provocando sérios prejuízos a população e aos cofres públicos. Uma proposta adotada pela maioria das distribuidoras é elaborar mapas de todas as redes de distribuição, sendo através de mapas fornecidos por algumas empresas, por verificações direta ou investigações indiretas. Estas últimas vêm sendo amplamente utilizadas devido ao seu caráter não invasivo no subsolo. Dentre as investigações indiretas, utilizadas em ambiente urbano, destacam-se as geofísicas, representadas, principalmente, pelos métodos GPR (Ground Penetrating Radar), eletromagnético indutivo (slingram) e elétrico (eletrorresistividade). Apesar da eficiência destes métodos, algumas imprecisões, provocadas principalmente pela heterogeneidade do subsolo, permanecem. Uma maneira de eliminar estas incertezas é a realização de testes em áreas onde se tem conhecimento das propriedades físicas do alvo e do subsolo. Entre as principais áreas de testes geofísicos conhecidas, destacam-se: a do LCPC (Laboratoire Central des Ponts et Chaussees) em Nantes, França (Grandjean et al., 2000); o Stanford University Environmental Test Site (SUETS) da Stanford University,
USA (in Reynolds, 1997; Salem et al., 2002); e a área de testes do Departamento de Geociências da Western Michigan University, USA (Sauck et al., 2002).

A partir de 2002, o Prof. Dr. Jorge Luis Porsani, com o apoio financeiro da FAPESP (Fundação de Amparo a Pesquisa do Estado de São Paulo), iniciou o projeto de implantação da primeira área de testes de geofísica rasa do Brasil, o SCGR (Sítio Controlado de Geofísica Rasa) do IAG-USP (Porsani, 2002; Borges, 2002). O SCGR consiste em uma área de $1500 \mathrm{~m}^{2}(30 \mathrm{~m} \times 50 \mathrm{~m})$ dentro do campus da Universidade de São Paulo, na capital (Figura 1). Nesta área, ao longo de sete linhas, foram implantados vários tipos de objetos e materiais, tais como: manilhas de concreto, tubos metálicos e de PVC, cabos elétricos, tambores metálicos e plásticos, caixas de areia e brita, potes cerâmicos, paredes de tijolos, entre outros. Estes materiais foram enterrados visando simular situações reais de ambientes urbanos. $\mathrm{Na}$ área predominam sedimentos argilosos da planície de inundação do rio Pinheiros, sobrepostos por sedimentos argilo-arenosos dispostos na área para aplainamento do terreno (aterro).

Para caracterizar o subsolo (background) antes da implantação dos alvos dentro do SCGR foram realizadas investigações geofísicas com os métodos GPR, eletrorresistividade, eletromagnético indutivo e magnético, visando identificar a estruturação do aterro, heterogeneidades físicas e a interface aterro versus paleossolo. Neste trabalho serão apresentados alguns dos resultados das aquisições geofísicas de tomografia elétrica (TE) realizadas antes da implantação do SCGR.

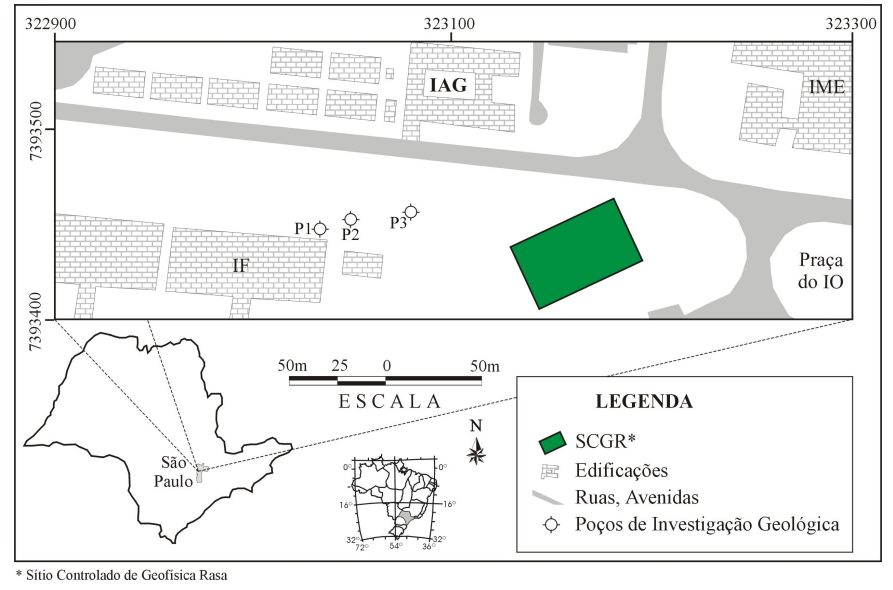

Figura 1 - Mapa de localização da área do SCGR do IAG dentro do campus da USP, São Paulo. 
Utilização de tomografia elétrica na identificação da interface entre uma cobertura antrópica e sedimentos fluviais na área do Sítio Controlado de Geofísica Rasa do IAG/USP

\section{Metodologia}

Os dados de tomografia elétrica 2D foram adquiridos com o arranjo dipolo-dipolo devido ao melhor acoplamento eletromagnético, possibilitando assim correlacionar com maior precisão dados de polarização induzida (IP) com dados de resistividade aparente (Ward, 1990). Apesar da diminuição do potencial medido com o distanciamento dos dipolos, uma voltagem de 200 Volts utilizada foi 0 suficiente para se obter bons resultados. Foram adquiridos 51 perfis de tomografia elétrica ao longo do SCGR. Os perfis foram realizados na direção N-S magnético, com espaçamento entre os dipolos de $1 \mathrm{~m}$ e 7 níveis de investigação. A Figura 2 ilustra a disposição dos pontos de investigação obtidos com a técnica do Caminhamento Elétrico (CE) ao longo de uma linha do SCGR.

O equipamento utilizado foi o Syscal R2, fabricado pela empresa francesa IRIS, de propriedade do Departamento de Geofísica do IAG/USP. Para diminuir o tempo de aquisição de cada perfil, foi utilizado um comutador eletrônico de eletrodos desenvolvido no Laboratório de Instrumentação Geofísica do Departamento de Geologia do IAG/USP (Hiodo et al., 2001). Com a utilização deste comutador e de um sistema de conexão entre o eletrodo e o cabo de potencial foi possível diminuir o tempo de aquisição de cada perfil de TE.

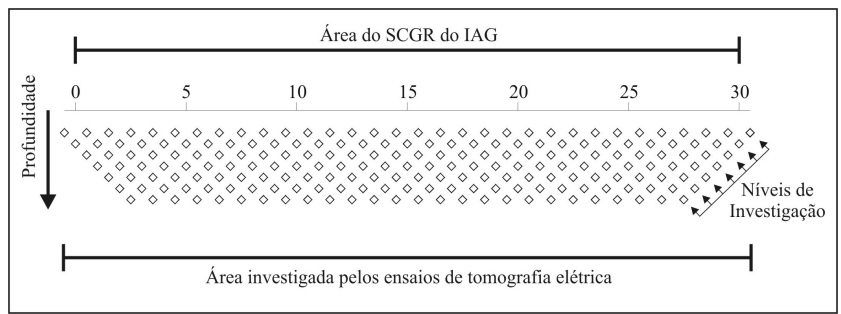

Figura 2 - Disposição espacial dos pontos de investigação de tomografia elétrica dentro da área do SCGR

Os dados de TE foram divididos em nível de investigação e agrupados para elaboração de mapas de níveis de resistividade aparente. Algumas seções foram modeladas através do software Res2dinv (Loke, 1999) visando determinar a profundidade limite do aterro dentro da área do SCGR-IAG.

\section{Resultados}

Nos mapas de resistividade aparente apresentados em níveis (Figura 3) percebe-se uma anomalia condutiva na parte leste do SCGR, quanto mais profundo é o nível maior é a condutividade. A partir do nível 5 ela se torna constante, ou seja, aparece em todo o sítio, definindo uma única camada.

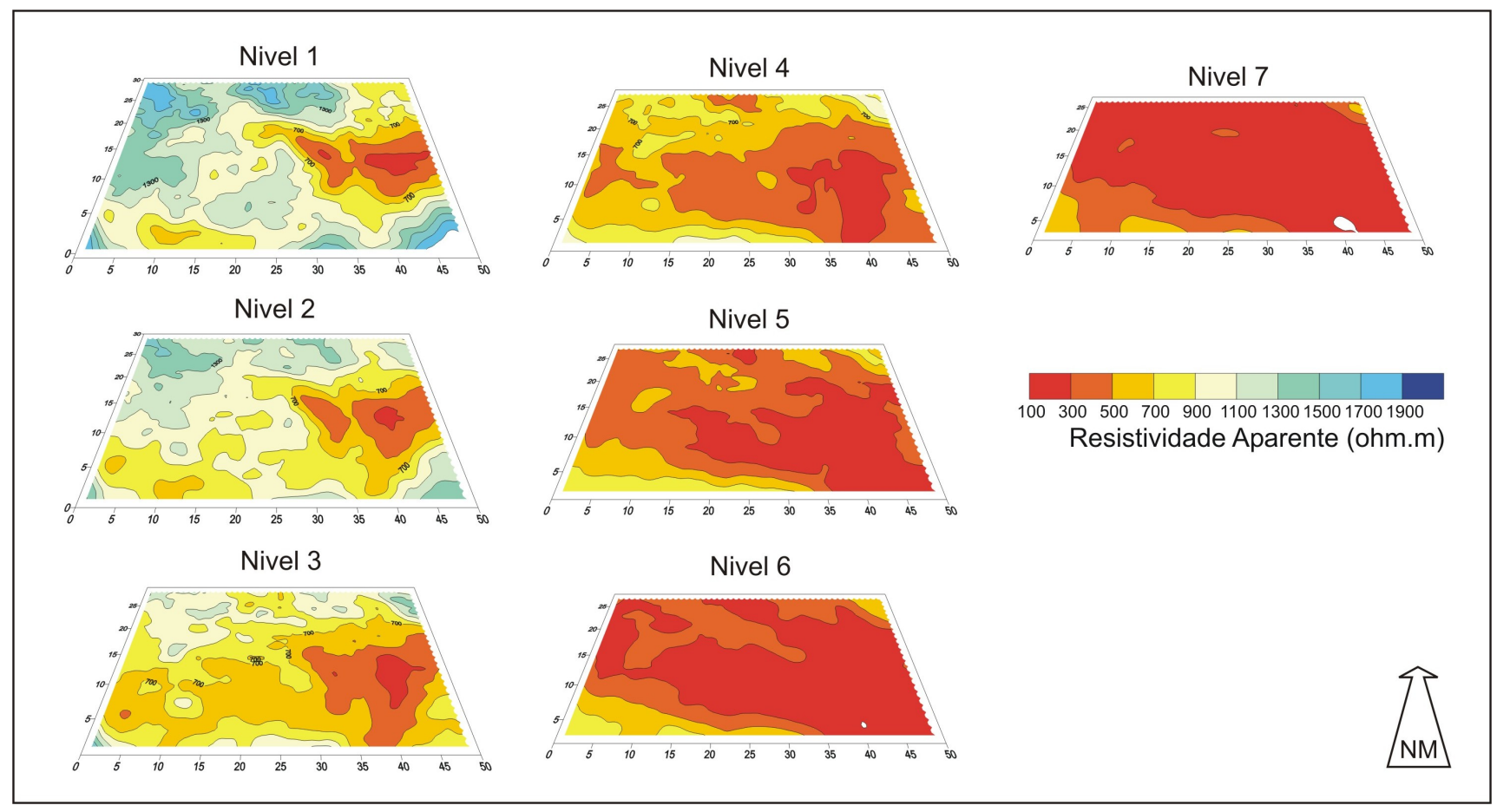

Figura 3 - Mapas de isovalores de resistividade aparente dos níveis de investigação da área do SCGR-IAG obtido através da técnica do caminhamento elétrico com o arranjo dipolo-dipolo. Nota-se uma zona condutiva na parte leste da área nos níveis 1 a 4 . O nível 5 adiante define os valores de resistividade aparente do paleossolo. 
A Figura 4 mostra a pseudosecção de resistividade aparente da Linha $27 \mathrm{~ns}$, com os dados obtidos em campo (a), a pseudosecção sintética (b), e o modelo resultante da inversão (c). No modelo observa-se que há um aumento no valor da resistividade aparente, da parte superior, na direção norte (em sentido a $30 \mathrm{~m}$ ). O modelo mostra um corpo resistivo mergulhando suavemente na direção norte, ou seja, a camada resistiva apresenta um espessamento da posição $0 \mathrm{~m}$ para $30 \mathrm{~m}$. Utilizando o limite de resistividade de 400 ohm.m para camadas argilosas (Telford et al., 1990), pode-se determinar, através do modelo geolétrico obtido, que a camada de aterro possui uma espessura, variando entre $1,2 \mathrm{~m}$ a 2,10 $\mathrm{m}$.

\section{Discussão e Conclusões}

A zona condutiva identificada nos dados de tomografia elétrica indica um aumento na concentração de argila na parte leste do SCGR, e define o limite entre o pacote de material antrópico (aterro) e o topo dos paleosedimentos argilosos do rio Pinheiros.

Os resultados indicam que há um aumento na espessura do aterro na direção norte da área, conforme verificado nos dados geológicos obtidos com a escavação de trincheiras executadas na área (Figura 5).

\section{Agradecimentos}

Os autores agradecem a Fundação de Amparo a Pesquisa do Estado de São Paulo (FAPESP) pelo apoio financeiro, processos $n . .02 / 07510-0$ e n. $02 / 07509-1$. Ao IAG-USP pela oportunidade de desenvolver esta pesquisa. Ao Instituto de Física da USP por ceder a área para implantação do SCGR. Ao técnico Ernande e a mestranda Selma pelo auxílio na aquisição dos dados.

\section{Referências}

Borges, W.R., 2002. Caracterização geofísica de alvos rasos com aplicações no planejamento urbano e meio ambiente: estudo sobre o sítio controlado do IAG/USP. Projeto de Pesquisa de Doutorado, Departamento de Geofísica do IAG-USP. Processo FAPESP No. 02/075100, São Paulo - SP.

Grandjean, G., Gourry, J.C. \& Bitri, A., 2000. Evaluation of GPR techniques for civil-engineering applications: study on a test site. Journal of Applied Geophysics, Elsevier, 45, 141-156.

Hiodo, F. Y.; Silva, N.; Elis, V. R.; Galhardo Filho, L. \& Silva, J. E. da. 2001. Projeto de um sistema semiautomatizado de medidas de potencial elétrico natural ou induzido do solo para aplicações geolétricas. In: Anais do 7.․․ International Congress of The Brazilian Geophysical Society, Salvador, BA, p. 136-139.

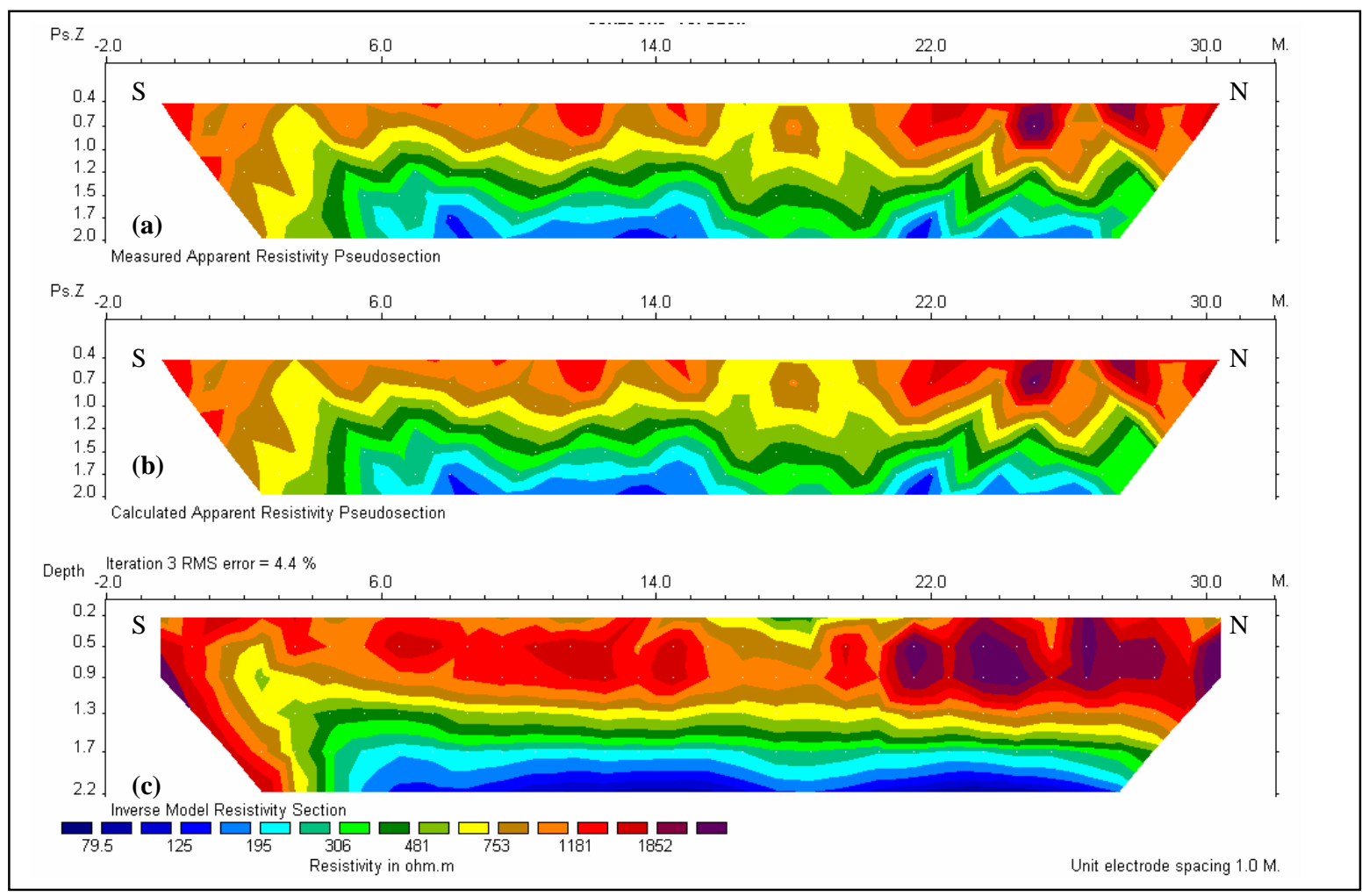

Figura 4 - (a) Pseudosecção de resistividade aparente dos dados de campo. (b) Dados sintéticos do ajuste do modelo. (c) Modelo de resistividade obtido após inversão. Nota-se um aumento da espessura do corpo resistivo superior em direção a norte. 
Utilização de tomografia elétrica na identificação da interface entre uma cobertura antrópica e sedimentos fluviais na área dq Sítio Controlado de Geofísica Rasa do IAG/USP

Loke, M.H., 1999. Res2Dinv versão 3.4 for Windows 3.1, 98 and NT. Rapad 2D Resistivity \& IP Inversion using the least-squares method. Software Manual. Disponivel em: inttp: //www.abem.sé

Porsani, J.L., 2002. Caracterização geofísica de alvos rasos com aplicações no planejamento urbano, meio ambiente e arqueologia: estudo sobre o sítio controlado do IAG/USP. Projeto de Auxílio à Pesquisa. Processo FAPESP No. 02/07509-1, São Paulo - SP.

Reynolds, J. M. 1997. An Introduction to Applied and Environmental Geophysics. John Wiley \& Sons, New York, NY, USA, 796p.

Salem, A.; Ravat, D.; Gamey, T. J.; Ushijima, K., 2002. Analytic signal approach and its applicability in environmental magnetic investigations. Journal of Applied Geophysics, Elsevier, 49: 231-244.
Sauck, W.A., 2002. Controlled Site for Shallow Geophysics. Institute for Water Sciences. Department of Geosciences. Western Michigan University. Disponível

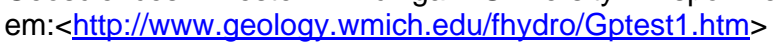
acessado em 20/04/2002.

Telford, W. M.; Geldart, L. P. \& Sheriff, R. E. ,1990. Applied Geophysics. Second Edition, Cambridge University Press, United Kingdom, 770p.

Ward, O.S.H., 1990. Resistivity and Induced Polarization Methods. Geotechnical and Environmental Geophysics, Volume 1: Review and Tutorial. Society of Exploration Geophysics, Tulsa, OK, pp. 147-189.

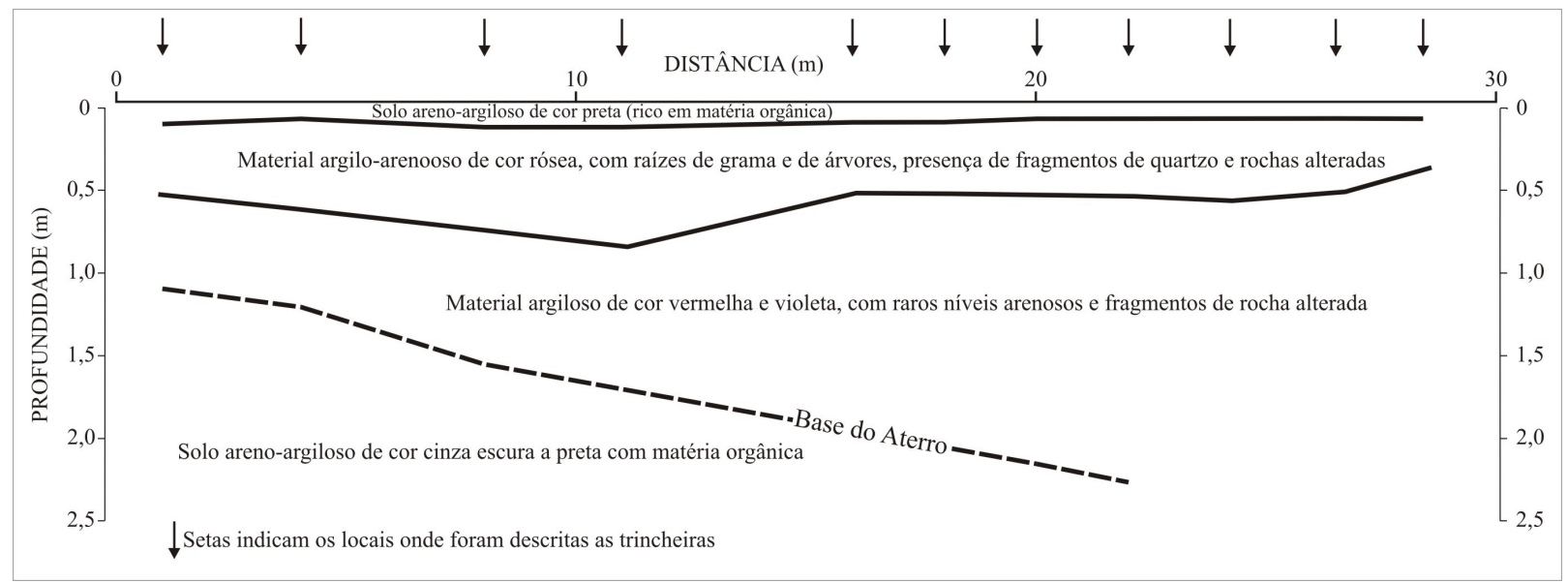

Figura 5 - Seção geológica da Linha 27ns, elaborada a partir de dados geológicos das trincheiras escavadas para enterrar os alvos, mostrando as principais interfaces geológicas. 